\title{
Exploring Social Interactions and Attributes of Casual Multiplayer Mobile Gaming
}

\author{
Kevin A. Li \\ University of California, San Diego \\ 9500 Gilman Dr. \\ La Jolla, CA 92092, USA \\ k2li@cs.ucsd.edu
}

\author{
Scott Counts \\ Microsoft Research \\ One Microsoft Way \\ Redmond, WA 98052, USA \\ counts@microsoft.com
}

\begin{abstract}
We explored the social and gaming attributes of interactive casual gaming on mobile phones. To do so, we developed a game called MoMENTus, a multiplayer mobile brain teaser game, and deployed it to 43 participants, divided into various multiplayer and team configurations, over the course of one week. Participants generally liked the interactive and mobile aspects of our casual game, while the team concept was unexpectedly less compelling. Our results suggest that mobile multiplayer casual gaming is a promising new direction, with game play fostering a feeling of community among players. Team play was found to be counter to the casual nature of the game, but competition acted as an effective motivator. Additionally, since mobile phones are "always-on-person," casual mobile gaming was successful in filling interstitial time. Findings address aspects of multiplayer mobile casual gaming, such as the role of competition, manner in which these games are played, and the impact of the type of content on such games. Implications for the design of interactive casual games for mobile phones are discussed.
\end{abstract}

\section{Author Keywords}

\section{ACM Classification Keywords}

H5.m. Information interfaces and presentation (e.g., HCI): Miscellaneous. K.8.0. Personal Computing-games.

\section{INTRODUCTION}

Traditionally, the gaming industry has focused on creating immersive environments, driven by advances in graphics and computing power. Many commercial games for mobile phones take advantage of these technical advances, employing rich graphics in their games. Research efforts in

Permission to make digital or hard copies of all or part of this work for personal or classroom use is granted without fee provided that copies are not made or distributed for profit or commercial advantage and that copies bear this notice and the full citation on the first page. To copy otherwise, or republish, to post on servers or to redistribute to lists, requires prior specific permission and/or a fee. MC'07 (Mobility'07), September 10-12, 2007, Singapore.

Copyright 2007 ACM 978-1-59593-819-0_.....\$5.00 this domain have largely been geared towards augmented reality, attempting to merge the physical world with a virtual world.

Recently, casual games have become increasingly popular $[3,4]$. However, few multiplayer mobile casual games exist and little work has been done observing the social aspects of such casual gaming. While some work has been done with location-based gaming on mobile platforms, these tend to more active than casual in that they require the user to change their behavior as part of game play. Given that mobile phones are an always-carried device, they provide an excellent platform for applications that leverage interstitial time such as when waiting for a friend or sitting on the bus. This ubiquity, combined with recent advances in mobile phone graphics capabilities and the nature of mobile phones as communication devices, points to the mobile phone platform as an ideal vehicle for causal interactive gaming.

While mobile phones seem like an obvious platform for multiplayer casual games, a number of questions regarding social usage arise. How might playing with known vs. unknown players impact fun and social consequences? Would these effects vary between individual and team play conditions? Additionally, with casual games being of a very different nature compared to immersive games, in what ways might these differences translate into design implications? Finally, it would be interesting to observe the social effects that playing a multiplayer casual game might have on intra-group communication. Could a multiplayer casual game support social connectivity in a way that SMS messages have been shown to?

To explore these questions, we designed, deployed, and studied the usage of a casual, interactive game for mobile phones. The primary contribution of this paper, and our motivation for this work, is an initial examination of these socially oriented questions surrounding casual, interactive games for mobile devices. We developed a rich client multiplayer casual game for mobile phones running Windows Mobile, using SMS as a means for sending data to and from a centralized server. We chose SMS over a GPRS-based internet protocol given the considerable gap between data services and SMS adoption $[1,2]$. The game 
was deployed to 43 people who played a multiplayer brain teaser game over the course of one week. Presented below, following a discussion of related works, is a description of our game and results from this field deployment. High level findings suggest multiplayer mobile casual gaming as a strong alternative to single player casual gaming. Our discussion section presents a number of secondary findings and implications for design that emerged from usage patterns and observations of the different multiplayer and team game play conditions.

\section{RELATED WORKS}

While there has been relatively little work on the social aspects of casual multiplayer gaming on mobile devices, there has been significant work done in the areas of multiplayer gaming environments and mobile phone gaming, as well as different types of mobile device-based social communication.

\section{Multiplayer gaming}

Early work with multi-user dungeons (MUDs) has shown that a rich, immersive environment is not necessary for a compelling social experience [14]. The social aspects of MMORPGs have been studied more extensively in recent times, thanks to their increased popularity. Ducheneaut et. al. have done significant work in this area, finding that gaming has many social aspects, often with implications beyond those of the game $[16,17]$. Additionally, while some games such as Star Wars Galaxies (SWG) have been designed to support social interaction between players, they have met with mixed success. One the one hand, a rich interface does not necessarily translate to increased social interaction, as they can be "over-designed" for this purpose $[18,23]$. However, creating interdependency between players clearly increases social interaction; some games explicitly require social coordination to achieve game goals [20].

One interesting finding among MMOGs is the "alone together" phenomenon whereby it is not through the explicit interaction between players that has social consequences, but simply the act of being proximal to other players that provides the compelling social factor [15]. This finding has promising implications for a gaming world where text communication with other players might not be as convenient as typing messages on a keyboard, such as on a mobile phone.

Another interesting finding from MMOG social research has found that people in these online worlds are more likely to be more social with other players when they belong to groups, compared to non-group associated players [7]. This suggests there may be social interaction differences between game configurations where players know each other beforehand versus configurations where players do not know each other a priori. The idea of asynchronous casual multiplayer gaming has been proposed before, but in less interactive forms. These types of games involve waiting for other players to take their turns, similar to board games, significantly lowering interactivity levels [12]. While this approach attempts to appeal to casual gamers, it seems to lack the flavor of social interaction that would likely to arise from simultaneous play.

\section{Mixed-Reality Gaming}

Mixed-reality gaming has been a compelling topic of interest for some time as it is clearly a rapidly growing market. Thanks to GPS devices and recent advances in mobile location aware computing, many mobile games have been able to explore new paradigms involving location [21]. Augmented reality games that merge the physical and virtual world have been an exciting consequence made possible by these advances in context sensing. While a complete survey of all existing mobile games is beyond the scope of this paper, some of the more representative works are described here.

Can You See Me Now? and Uncle Roy All Around You are mixed reality games that explore augmented reality as well as the corresponding social aspects $[11,18]$. In studies of these games, context was shown to be a consequence of social factors as opposed to technical ones, illustrating the importance of social structure in mobile games. These types of games clearly required a player's attention for a long period of time.

A somewhat more casual game, also geared towards social interaction is the STARS platform for hybrid computeraugmented table-top games [22]. The idea behind this project is to preserve the rich social interaction of traditional board games, while adding the power of technology. While an interesting concept, it does not scale to the mobile platform.

Finally, a number of games have been developed with network connectivity in mind. Treasure involves movement through wi-fi networks, where users collect virtual coins scattered in the environment, using their PDAs [8]. Similarly, the Feeding Yoshi game [9] examines issues of "seamful design" in mobile applications with a game in which actions of a PDA-based character depend on the coverage and security of wireless network nodes in the surrounding environment. Real Tournament is another mobile augmented reality game [24]. In this multiplayer game, a player's physical orientation gets mapped to a virtual world using sensor data from a mobile device equipped with a sensor array. These mixed-reality games take advantage of advances in location-aware computing but have not examined the social aspects of casual mobile gaming. While we considered examining a location-aware game, placing physical requirements on players seemed counter to the casual gaming focus of our investigation.

\section{Mobile Social Communication}

Text messaging has become wildly popular as a means of social communication, with a variety of uses from couples and close friends using it to "stay connected" throughout 
their days [19] to teenagers using text messaging as a form of gift giving [1]. These uses of text messaging suggest a usage scenario where messages are sent not to convey information, but rather to stay in touch with others. Could a similar effect be observed in a multiplayer game played via mobile phones?

Other mobile social applications exist for the sole reason of connecting folks to those around them. Examples of services for helping friends keep in touch include Dodgeball, where users can check in to report their time and location, thus adding social coordination [5], and Plazes, which helps mobile users connect to other people with similar interests [6]. Slam is another social mobile application that investigates group-based interaction through messaging and photo sharing, reporting on how such a communication paradigm might facilitate social interaction [13].

While a number of social mobile applications exist, they are all centered towards explicitly reaching out to other people with messages or phone calls. Can a multiplayer game foster social connectivity with casual game play over an extended period of time?

\section{MOMENTUS DESIGN}

MoMENTus is an interactive multiplayer brainteaser game designed for Smartphones and PDAs running Windows Mobile 2003/5.0. Unlike most other research in this space, the contribution of this work is to examine social questions surrounding casual mobile gaming, rather than to present a novel mobile game. The game rules are described briefly here, followed by a description of the system architecture.

\section{Game Rules}

The goal of MoMENTus is to get as many points as possible and take over the brain by solving brain teasers. To facilitate this process, the brain has been divided into 6 navigable regions (Fig. 1). Each region contains a different type of brain teaser. Players can select different regions by using the directional pad on their mobile device. After selecting a brain region, players are presented with a puzzle (Fig. 2). Each attempt to solve a puzzle costs 1 turn.

Turns are accrued on system updates, which occur 4 times per day, at 6 hour intervals. On an update, players receive an SMS message informing them that an update has occurred as well as the number of turns they have remaining. Turns never expire but there is a cap on the number of turns one can save up, approximately equal to the number of turns that would accrue over two days of inactivity. Also, game update information is sent to the phone specifying what player has solved which puzzles. These game update SMS messages have a special game header and are intercepted by the application and processed accordingly. Leaderboard statistics are updated with new point totals and region shading is updated as well. Regions are shaded based on the number of puzzles solved by the team/player closest to conquering the region.
Teams/players receive 1 point for every puzzle solved and 6 bonus points for taking a region by solving all of that region's puzzles first. The winner is the person and/or team with the most points at the end of the game. A leaderboard screen (Fig. 3) shows team and player standings. This feature was introduced to provide social visibility to prevent social loafing, whereby a team member does not contribute to the team since they are not held socially accountable. MoMENTus also supports in-game SMS messaging between players (Fig. 4).

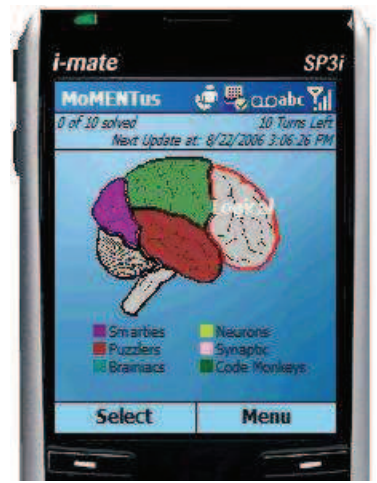

Figure 1. Main Screen.

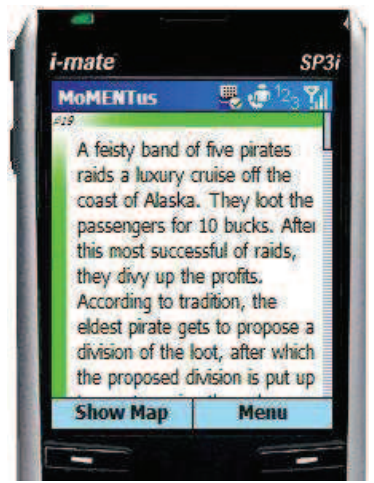

Figure 2. Sample Puzzle.

\section{System Architecture}

To support MoMENTus game play, a client-server architecture was developed, leveraging SMS as a transport protocol. While many mobile phones on the market are capable of using GPRS connections for sending and receiving data, the majority of subscribers do not have data plans while many customers do have SMS messages as part of their monthly plan [1]. To utilize SMS messages for game update information, an application layer was written on top of SMS.

All backend web services run from a server running Windows Server 2000. Database queries are then performed through this server, connecting to a SQL Server database on a different machine. To handle SMS message routing, 4 Smartphones running Windows Mobile 5.0 are each connected to a different PC using a USB cable and Activesync. Webservice calls are then invoked from these PC machines as necessary.

To communicate with the backend server, the client sends an SMS in the game data format (a text message prepended with "\#") to one of the 4 server phones described above. The phone forwards incoming messages to the $\mathrm{PC}$ it is attached to, which then calls a Webservice method, passing the SMS message payload along. Game updates are sent out from these phones as well. A round robin queuing policy is used for load balancing to handle the failure of a server phone and to deal with surges in activity. 
Although the focus of this work was on the social side of game play, we feel the pairing of a rich client interface with an SMS transport a combination worth noting. In addition to the aforementioned paucity of GPRS data plans, in many countries around the world, particularly in developing areas, SMS is the primary mobile communication protocol. We found this SMS-based approach raised a number of technical issues that, while outside the scope of this paper, are worth a brief mention.



Figure 3. Leaderboard.

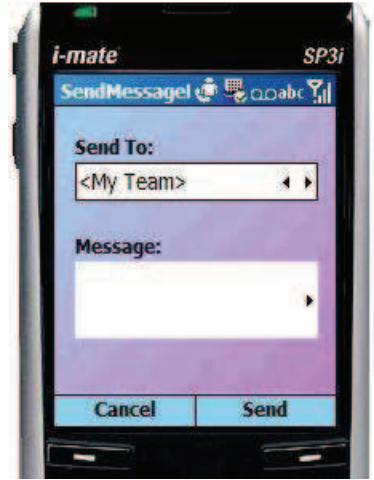

Figure 4. Messaging Window.

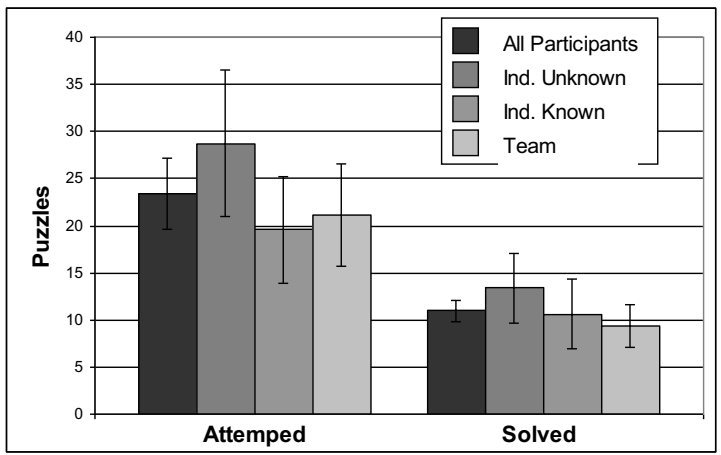

Figure 5. Puzzles attempted and solved.

How do you support an interactive system when each transmission costs the end-user money? Ideally, system updates would be performed each time a client changes state. However, this is unfeasible when sending/receiving SMS messages has cost associated with each message. In an attempt to reduce the number of sent/received SMS messages, synchronization of game state becomes an issue. This can be partially circumvented by (1) requiring clients to only submit local changes and (2) requiring the server wait until some number of client updates are received before sending out a system update. While this works fairly well most of the time, there are instances when update messages get lost or delayed, resulting in clients using stale data, which can be especially problematic when clients display inconsistent information among teammates. These issues were identified and deemed as acceptable given the casual nature of the game.

\section{EXPERIMENTAL FIELD DEPLOYMENT}

An experimental field study was conducted to assess both the social and game design aspects of our casual, interactive mobile game. 43 participants were recruited via corporate email distribution lists, divided into experimental conditions described below, and played MoMENTus over the course of 1 week. The only requirements for participating were ownership of a Smartphone and an interest in playing brainteasers. Participant demographics included ages ranging from 21 to 53 , and 39 men and 4 women, which was less gender balanced than was desired, but the result of available and willing participants. 36 participants were Caucasian, 7 Asian-American.

\section{Study Design}

The study employed a $2 \times 2 \times 1$ design, with a known versus unknown variable crossed with a multiplayer versus team variable (Table 1). The known/unknown variable indicated whether the game players knew one another prior to playing the game. The multiplayer/team variable defined whether participants played individually, but against one another or on teams. An additional "solo" condition was included in which participants played the game by themselves. Participants were randomly assigned to one of the five conditions except in the two known conditions where it was necessary to ensure that the participants did know one another. Although we were interested in usage and subjective experience measures across all participants, we hoped these conditions would shed light on a few of the social (e.g., playing with people you know vs. with strangers) and game design elements of casual, interactive gaming (e.g., playing on a team as opposed to by yourself).

The five resulting conditions were as follows:

\section{Solo}

Participants played the game by themselves.

\section{Known/Unknown Multiplayer}

Participants played by themselves, and against one another.

\section{Known/Unknown Team}

Participants played together in teams of $2-4$ that competed against each other. Known condition participants knew their teammates but not their opponents.

\begin{tabular}{|c|c|c|c|}
\hline & Multiplayer & Team & Solo \\
\hline Unknown & 11 & 8 & \multirow{2}{*}{6} \\
\cline { 1 - 2 } Known & 6 & 12 & \\
\hline
\end{tabular}

Table 1. Number of participants in social configurations. 


\section{Game Play}

As mentioned, participants had one week to play MoMENTus. Prior to beginning their game, participants completed a short questionnaire so we could collect demographics as well as gaming and mobile device history information. Participants then proceeded to play MoMENTus for one week as described above: solve as many puzzles and take as many brain regions as possible within the bounds of the daily turn allocation scheme. Reminder text messages were sent out four times per day that reported current game standings. To finish the study, participants completed a second questionnaire.

\section{Data Collection}

Three types of data were collected. Usage statistics, both on the game server (e.g., how many puzzles were solved) and on individual client devices (e.g., how many times the leaderboard was checked), to assess player interaction with various parts of the game. The questionnaire data were utilized to assess players' subjective experience of playing the game, such as how fun the game was, as well as social aspects of game play like how connected to other players they felt. Finally, a few participants were interviewed for qualitative feedback.

\section{STUDY RESULTS}

\section{Usage}

As indicated above, participants were semi-randomly assigned to one of five game play conditions. A first finding regarding usage was that 17 participants did not attempt even a single puzzle. On the face this is a discouraging result, but closer inspection revealed that the distribution of these participants was far from equal across the game play conditions. In fact, six of them were in the solo condition. Six of the remainder participants were in the unknown team condition. The other three conditions saw only a person or two fail to participate.

Note that the data analyses do not include participants who did not attempt any puzzles, simply because they had no usage data to asses and to serve as the basis for questionnaire feedback. The unknown team condition was also dropped from analyses because it contained only two participants who attempted puzzles and completed the study questionnaires. Consequently the team condition shown in the figures reflects the known team condition.

Those participants that did attempt puzzles did so at an average of about 23 puzzles during the course of the game, or about 3 puzzles per day (Figure 5). Given that some puzzles were fairly difficult and thus time consuming, this number fits with the estimated half to full hour of game play per day on average that participants reported in the post-study questionnaire. That participants only solved just over 10 puzzles (or about 1.5 puzzles per day), indicates the challenging nature of the brainteasers. Participants in the individual unknown condition attempted and solved the



Figure 6. Game map and leaderboard usage.

most puzzles, although not significantly more than the other conditions.

To get a sense of how much participants interacted with the game beyond attempting and solving puzzles, we logged their interactions with the different game client screens. As shown in Figure 6, participants viewed the game map (the brain shown in Figure 1) 40 times (just under 6 times per day) and the leaderboard (Figure 3) 12 times (just under twice a day) on average. Again, those in the individual unknown condition interacted with the game more than participants in any other condition, although not at a statistically significant increase.

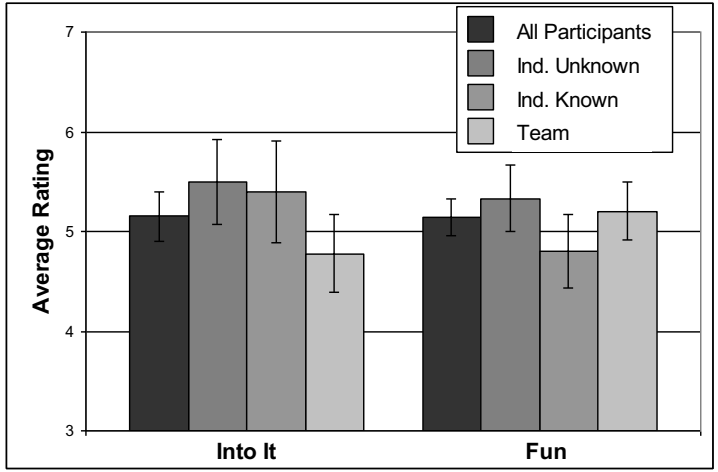

Figure 7. Game related questionnaire measures.

\section{Questionnaire Responses}

Upon completion of game play, participants filled out a questionnaire to capture their subjective experience of playing the game. Primary measures concerned how fun the game was and to what extent it became a part of their lives during the course of the study, as well as social aspects of the game. Figure 7 shows that participants on the whole found the game captivating (roughly 5 out of 7 on a scale ranging from $1=$ not at all to $7=$ very much), as well as fun (also about 5 of 7). Like both sets of usage measures, the individual unknown condition appeared to lend itself to the 
most compelling and fun game to play, but differences between conditions were not significant for these measures.

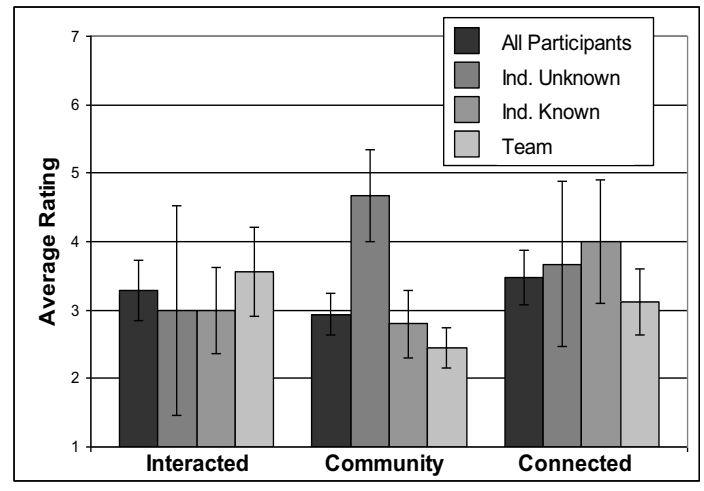

Figure 8. Socially oriented questionnaire measures.

Regarding social aspects of the game, participants generally responded below the midpoint of the rating scale. Figure 8 shows these ratings for our three main social measures: degree to which participants felt they interacted with others, felt they were part of a community, and felt connected to one another. Those in the individual unknown condition reported significantly higher responses for feeling like part of a community than any of the other conditions $(t=3.225$, $14, \mathrm{p}<.01)$. Although participants in the individual unknown condition did show more game activity and interest, this is somewhat surprising considering that those in the individual known and team conditions knew each other prior to the game and would thus be expected to report higher ratings on sense of community.

More reflective of the potential impact of casual multiplayer mobile games on social relationships is the change in social measures collected before and after playing the game. Table 2 shows a statistically significant $(\mathrm{p}<0.05)$ increase in the measure of "how well do you know your fellow game players" when collapsed across all participants. The second question, "how interested are you in a casual social activity with your fellow gamers such as a movie on a Friday night", did not show the same increases.

\section{QUALITATIVE FEEDBACK AND DISCUSSION}

\section{Game Elements}

A Constant Presence in People's Lives

The results provide insight into each element of the game: its intended casual and interactive nature, and its use of the mobile phone as a gaming platform. First, concerning the casual nature of the game, given the number of puzzles attempted ( 3 per day) and amount of time spent playing the game (half to full hour per day), the game seemed to have succeeded in becoming a casual activity to play here or there throughout the week. This is in contrast to a fully immersive console game that is generally played less frequently but for longer sessions. This does not mean the game failed to captivate its audience. Because the turn allocation scheme forced the puzzle attempts to be spread out over the course of the week, the total amount time spent playing (roughly $3.5-7$ hours) suggests this game was a consistent presence in people's lives throughout the duration of the game.

In fact, even beyond the actual time spent playing the game, participants remarked during the qualitative feedback sessions that they thought about the game even when not playing: the turn allocation scheme "added expectation and made you think about it when you weren't playing."

\section{Too Compelling?}

The casual nature of the game raises an interesting tension between keeping a game casual, but also compelling. On the one hand, obsession with a game is a success, yet also may not be the goal for the game because of the medium. Or should we consider mobile games that are obsessive to be successes if they have moved beyond casual gaming, at least in the sense of the amount of the gamer's mental attention appropriated? "For me I would think about it every day, and every few hours I would be checking in to see if I had more turns," one participant said in a follow up interview. Is every few hours too much for a casual game? This question becomes more important in the mobile context given the "always on" connectivity. Every few hours over the course of a week means players are checking in on the game at work, on the bus, in the evening, and so on, making it a substantial part of what they do. This double-edged sword issue would be exacerbated given a game more compelling than brain teasers. Nonetheless, a take-away point is that the combination of an ongoing and mobile device-based game yields considerable potential for becoming a significant focal point for end users.

\section{Always-on-Person}

This "reachability" of having the game on a mobile phone was also cited as a positive for the simple reason that users did not have to be at PC or gaming console to play. "To me having it on a phone was probably better because I didn't have to be at my computer desk. I would be on my couch and get the notice that 'hey I have more turns' and go for it," remarked one person. Several players described attempting puzzles in their cars, including staying in the car to play while his kids were running an errand in a store. This mobility advantage applies to existing mobile gaming platforms like the Sony PSP, but the connectivity to the game server and "always on person" nature of the mobile phone greatly enhances it. The person sitting on the couch only knew about his turns because he had his phone on him and it buzzed when it received a notification from the game server announcing additional turns.

\section{Social Interactions}

One goal for this research was to evaluate the impact of group-based interactions on mobile devices in a gaming context on social variables, such as how this medium can 
help people get to know one another. The results show an increase in feeling part of a community with a statistically significant increase in the 'how well do you know your fellow gamers' before and after question.

The most telling result concerning the critical role of adding interactivity is that no solo condition participants even played the game. Presumably they simply were not as interested as those assigned to an interactive version of the game. However, concluding that inclusion of a player interaction element will boost usage would be an oversimplification. For the multiplayer interactions to be successful they really need to be done right. For example, in MoMENTus, the team version of the multiplayer experience was of only moderate success. One participant who was on a team with another player who didn't participate as much as he did remarked that eventually he just started playing for himself: "there was only one other guy and he wasn't that good. [The teams] didn't matter that much because I was trying to max out my turns every time anyway."

\begin{tabular}{|c|c|c|c|c|}
\hline \multirow{4}{*}{ All } & $\begin{array}{c}\text { Know } \\
\text { Well }\end{array}$ & 4.52 & 5.19 & $<0.05$ \\
\cline { 2 - 5 } & $\begin{array}{c}\text { See } \\
\text { Movie }\end{array}$ & 4.85 & 4.95 & n.s. \\
\hline \multirow{2}{*}{$\begin{array}{c}\text { Individual } \\
\text { Unknown }\end{array}$} & $\begin{array}{c}\text { Know } \\
\text { Well }\end{array}$ & 1.33 & 2.67 & n.s. \\
\cline { 2 - 5 } Individual \\
Known & $\begin{array}{c}\text { See } \\
\text { Movie }\end{array}$ & 2.00 & 2.67 & n.s. \\
\cline { 2 - 5 } & $\begin{array}{c}\text { Know } \\
\text { Seel } \\
\text { Movie }\end{array}$ & 6.147 & 5.71 & 0.10 \\
\hline \multirow{2}{*}{ Team } & $\begin{array}{c}\text { Know } \\
\text { Well }\end{array}$ & 4.80 & 5.40 & n.s. \\
\cline { 2 - 5 } & $\begin{array}{c}\text { See } \\
\text { Movie }\end{array}$ & 4.89 & 5.22 & n.s. \\
\hline
\end{tabular}

Table 2. Social measures, before and after game.

\section{Team Play is Counter to Casual Gaming}

The potentially tenuous team dynamics raise several issues. First, the relative failure of the unknown team versions of the game (recall that only 2 people assigned to this condition actually attempted any puzzles) points to a social interaction issue regarding people who do not know one another having to work together. Difficulties with this arrangement may be due to participants feeling uncomfortable being on a team with people they didn't know, but also simply may be the result of competition being a better motivator. As one participant put it, "Team wasn't that great - If I were to design it, it would be individuals competing against one another. Getting stuck with a lame teammate kind of ruins it." This observation points to a second issue regarding teams and games of this type. There may be a fundamental tension between the team dynamic and the nature of casual games: players of casual games need to be able to play as much or as little as they want and the interdependencies of games may run counter to this critical attribute of casual gaming.

\section{Competition as a Motivator}

Opposite any shortcomings of the team conditions, was the trend toward the individual unknown version of the game working the best. That condition yielded significantly higher ratings on the 'I felt like a member of a community measure' and was slightly higher on the 'into it' and 'fun' measures and all usage measures. Again this suggests that competition is a good motivator for this type of game. This is good news for putting this type of game into practice, since finding players that know one another or facilitating logistical organization to get people onto a team together is considerably more effort than simply announcing a start time and letting people jump in to play against other players.

\section{Increased Communication}

Finally, a nuance of social interaction we also thought might be apparent was an increased level of digital communication through SMS messages and phone calls, especially in the known conditions. Surprisingly this was not the case, as players who knew each other would oftentimes communicate face-to-face instead. One participant remarked "theoretically, I would send messages, but I see him all the time and harass him verbally... also text isn't my favorite form of communication." This suggests that the multiplayer aspect of the game can actually increase communication between teammates, though not necessarily only via digital channels.

\section{Game Play and Design}

Filling Interstitial Time

Prior to deployment, we suspected the game would be played during the interstitial moments in participant's days, such as when on a bus. Follow up interviews supported this hypothesis. One participant remarked that he would play the game when "I have $10-15$ minutes of downtime when all I have is a phone, just whenever - an oil change, at a stoplight, whatever." Another participant commented on how he would play at work during his lunchtime or just when he needed a break from work. Other comments suggested that the game also saw more focused game play, as in the case of the participant that would sit on his couch at night and play for an hour or two in one session. This raised a frequently mentioned conflict between more challenging and interesting content that required additional game play infrastructure like a whiteboard or paper and 
pen, exceeding what could be done with the phone only. Some of the brainteasers required sketch space to solve, making them difficult to solve while sitting on the bus. The challenge for designers of mobile phone-based games is to generate engaging content that at least for some significant part of the game requires only the mobile handset for interaction.

One of the biggest complaints regarding team game logistics was the lack of a good indicator of what teammates had done in recent times. Which problems they had solved, their level of performance and their general participation level was important data for players. One participant commented that "it would be great to see what puzzles they had answered, etc." While our game had a leaderboard to keep players aware of game progress, it was difficult to tell how much progress had been made since the last update. A more animated interface could potentially address this, but would be somewhat difficult to implement since the mobile nature of the game makes it difficult to recognize when the user has time to give their full attention to the screen. Small display size also makes visualization of game progress difficult.

\section{FUTURE WORK}

While the study demonstrated significant differences between the conditions, a larger scale deployment would be useful for identifying both factors and effects that may be less pronounced and thus, not visible from the analysis performed here. Group dynamics might change with a single leader as opposed to all members being equal. Direct competition might also yield different results from the passive competition explored here. Also, larger group sizes would have been interesting to study but given recruiting constraints, this would have been difficult to deploy.

\section{CONCLUSION}

With the rapidly growing mobile gaming market and the increasingly popular casual game industry, interactive multiplayer casual games on mobile phones seem like an unexplored yet natural candidate for filling interstitial time. The results of deploying a multiplayer brain teaser game suggest multiplayer casual gaming on mobile phones to be a promising area, although with a few potential pitfalls, such as the relative ineffectiveness of team play. Yet multiplayer game play was successful with competition acting as a good motivator. Additionally, casual games on mobile phones opportunistically fill interstitial time thanks to the always-on-person nature of mobile phones.

\section{REFERENCES}

1. http://www.idc.com.

2. http://www.travellingwave.com/market_opportunity.htm.

3. http://seattlepi.nwsource.com/business/275609_casual28.htm.
4. http://seattletimes.nwsource.com/html/businesstechnology/20 03131656 btcasual17.html.

5. http://www.dodgeball.com.

6. http://www.plazes.com.

7. Axelsson, A.-S., Regan, T. How Belonging to an Online Group Affects Social Behavior - a Case Study of Asheron's Call. MSR Technical Report MSR-TR-2002-07.

8. Barkhuus, L. et al. Picking Pockets on the Lawn: The Development of Tactics and Strategies in a Mobile Game. In Proc. of Ubicomp 2005, pp. 358-374.

9. Bell, M. et al. Interweaving mobile games with everyday life. In Proc. of CHI 2006, pp. 417-426. ACM Press, 2006.

10. Benford, S., Magerkurth, C., Ljungstrand, P. Bridging the Physical and Digital in Pervasive gaming. Communications of the ACM, March 2005.

11. Benford, S., et al. The error of our ways: The experience of self-reported positioning in a location-based game. In Proc. of Ubicomp 2004.

12. Bogost, I. Asynchronous Multiplay: Futures for Casual Multiplayer Experience. In Proc. Other Players 2004.

13. Counts, S. Group-Based Mobile messaging in Support of the Social Side of Leisure. To Appear in JCSCW ??

14. Dourish, P. Introduction: The State of Play. In Proc. of CSCW 1998.

15. Ducheneaut, N., Yee, N., Nickell, E., Moore, R.J. 'Alone Together?' Exploring the Social Dynamics of massively Multiplayer Online Games. In Proc. of CHI 2006.

16. Ducheneaut, N., Moore R. J. Gaining more than experience points: Learning social behavior in multiplayer computer games. In Proc. of CSCW 2004.

17. Ducheneaut, N., Moore R. J. The Social Side of Gaming: A Study of Interaction Patterns in a Massively Multiplayer Online Game. In Proc. of CSCW 2004.

18. Flintham, M. et al. Where On-Line Meets On-The-Streets: Experiences with Mobile Mixed Reality Games. In Proc of CHI 2003. pp. 569-576. ACM Press, 2003.

19. Ito, M., Okae, D., Matsuda, M. Personal, Portable, Pedestrian. Mobile Phones in Japanese Life. MIT Press, Cambridge, MA, 2005.

20. Jakobson, M., Taylor, T.L. The Sopranos meets EverQuest: social networking in massively multiplayer online games. In Proc. of DAC 2003. pp. 81-90.

21. LaMarca, A., et. al. Place Lab: Device positioningusing radio beacons in the wild. In Proc. of Pervasive 2005.

22. Magerkurth, C., Engelke, T., and Memisoglu, M. Augmenting the virtual domain with physical and social elements. In Proc. of the International Conference on Advancements in Computer Entertainment Technology 2004. pp. 163-172. ACM Press, 2004.

23. Manninen, T. Interaction forms and communicative actions in multiplayer games. Game Studies, 3 (1). 2003

24. Mitchell, K., McCaffery, D., Metaxas, G., Finney, J., Schmid, S., Scott, S. Six in the City: Introducing Real Tournament - A Mobile IPv6 Based Context-Aware Multiplayer Game. In Proc. of the $2^{\text {nd }}$ workshop on Network and system support for games 2003. pp.91-100.

25. Taylor, A.S., Harper, R. Age-old practices in the new world: A study of gift-giving between teenage mobile phone users. In Proc. of CHI 2003. 